\title{
VÍNCULOS ENTRE MODELOS DE FINANCIACIÓN Y CATEGORIZACIÓN DE LOS SISTEMAS DE EDUCACIÓN SUPERIOR DE AMÉRICA LATINA
}

\section{Introducción}

La Reforma de Córdoba, de 1918, definió el modelo universitario en América Latina, cuyas características de autonomía, gratuidad y cogobierno contribuyeron decisivamente a la expansión de la matrícula, pasando de sistemas de elite a sistemas que democratizaron el acceso de nuevas clases sociales, las que, hasta ese momento, no eran consideradas prioritarias en los objetivos educativos.

No obstante, a fines de los 70, la masificación y la reducción del presupuesto público afectaron la capacidad de las instituciones para atender el crecimiento de la demanda, deteriorando la eficacia, eficiencia y equidad de la educación superior. Los países de la región pusieron en marcha importantes reformas que implicaron, entre otras cuestiones, la transformación de los modelos de financiación de las universidades públicas.

Estas medidas, aunque a ritmos diferentes, se han implementado en la gran mayoría de estos países, permitiendo a autores ${ }^{1}$ como Brunner (1994) y García (2002) la clasificación de los sistemas de educación superior (SES) latinoamericanos atendiendo fundamentalmente a su tamaño. Adicionalmente, Fernández y Fernández (2007) realizaron una nueva categorización mediante la aplicación de un análisis

1 Los autores han considerado para la clasificación de los SES las siguientes variables: Brunner (1994): matrícula total, tasa bruta de escolarización, número de instituciones y masificación de los SES; García (2002): matrícula total, y Fernández y Fernández (2006): matrícula total, ratio alumnos por profesor, gasto público educativo destinado a educación superior y PIB per cápita. 
cluster, considerando no sólo la dimensión de los SES, sino también otros criterios con un marcado carácter financiero, como son el gasto público educativo destinado a educación superior y el Producto Interno Bruto (PIB) per cápita, dando como resultado cinco grupos o clusters.

El objetivo de este trabajo es analizar los modelos de financiación de diferentes países latinoamericanos (México, Chile, Bolivia, Costa Rica y Uruguay), uno dentro de cada grupo de la clasificación propuesta por Fernández y Fernández (2007), con el fin de verificar si existen diferencias que puedan ser interpretadas como un rasgo característico adicional de los grupos resultantes. Dado que la categorización tiene en cuenta variables financieras, tales como el porcentaje de gasto público educativo destinado a la educación superior y el PIB per cápita, cabría esperar que aquellos países que destinan un menor porcentaje de gasto público a este nivel educativo, empleen modelos de financiación en que primen criterios de mercado. Por el contrario, aquellos en los cuales la participación pública es importante, posiblemente funcionen con modelos menos orientados hacia el mercado y el sector privado.

La literatura no ofrece hasta el momento un análisis profundo y comparado de los modelos de financiación de los SES de la región², por lo que nuestro aporte contribuye, en alguna medida, a conocer la evolución reciente de sus mecanismos financieros. Se ha empleado como metodología el estudio de casos, una estrategia interesante cuando no se dispone de suficientes datos que permitan analizar el contexto y los procesos implicados en el objeto de estudio. Por ello, previamente se analizaron separadamente los modelos de financiación de cada uno de los países. En esta investigación solamente reflejamos una síntesis comparativa de ese trabajo previo.

Este trabajo se estructura en cuatro secciones. En la primera se analiza la evolución de los SES, aportando algunas cifras sobre el

2 En un trabajo previo se realizó una comparación de los países que conforman el Grupo 1 "SES de gran tamaño" (Argentina, Brasil y México) (Fernández y Fernández, 2004). 
268 MODELO DE FINANCIACIÓN EN LOS SES DE AMÉRICA LATINA - Susana Fernández, Sara Fernández

crecimiento de la oferta y la demanda, lo que permite contextualizar el entorno donde se aplican los modelos de financiación. En la segunda, se considera el esfuerzo financiero realizado por los países en la financiación de la educación superior. En el tercer apartado se analiza la financiación de las universidades públicas, centrando la atención en los modelos utilizados para asignar los recursos públicos y en los mecanismos que incentivan la diversificación de fondos. Finalmente, se resumen las conclusiones más importantes del presente trabajo.

\section{El contexto de los SES: demanda y oferta}

El estudio de las cifras que describen el crecimiento de la matrícula y del número de instituciones de educación superior (IES) durante la última década sirve para conocer las razones que justifican la transformación de los modelos de financiación de la educación superior en los países analizados.

\section{Crecimiento de la demanda}

La matrícula de educación superior en América Latina ha evolucionado de forma distinta según las condiciones y características de cada país. Sin embargo, el crecimiento de la demanda se atribuye, en general, a la explosión demográfica en la década de los cincuenta, la masificación de los graduados de la secundaria y la competencia en los mercados laborales. De este modo, la matrícula pasa de 7,2 millones de estudiantes, en 1994, a 13,9 en 2003, lo que representa tasas brutas de escolarización (TBE) del 17,5\% y 28,7\%, respectivamente (ver tabla 1).

Tabla 1: Evolución de la matrícula de educación superior (1994-2003)

\begin{tabular}{l|r|c|c|c|c|c}
\hline \multirow{2}{*}{ SES } & \multicolumn{2}{|c|}{1994} & \multicolumn{2}{c|}{1998} & \multicolumn{2}{c}{2003} \\
\cline { 2 - 7 } & Total & TBE & Total & TBE & Total & TBE \\
\hline México & 1.420 .461 & 15,0 & 1.837 .884 & 18,7 & 2.322 .781 & 23,9 \\
\hline Chile & 327.074 & 26,6 & 406.553 & 33,8 & 567.114 & 46,2 \\
\hline Bolivia & 154.040 & 22,8 & 230.494 & 31,2 & 312.769 & 38,6 \\
\hline
\end{tabular}




\begin{tabular}{l|r|r|r|r|r|c}
\hline $\begin{array}{l}\text { Costa } \\
\text { Rica }\end{array}$ & 83.608 & 27,9 & 119.854 & 35,0 & 170.423 & 43,3 \\
\hline Uruguay & 74.842 & 29,8 & 86.231 & 32,1 & 105.268 & 40,5 \\
\hline $\begin{array}{l}\text { América } \\
\text { Latina }\end{array}$ & 7.521 .394 & 17,5 & 9.664 .574 & 21,0 & 13.934 .113 & 28,7 \\
\hline
\end{tabular}

Fuente: Elaboración propia a partir de González (2006).

El caso de México resulta llamativo, ya que cuenta con una matrícula que supera en 2003 los 2,3 millones de estudiantes, conformando uno de los países que cuentan con SES de gran tamaño (Brunner, 1994; García, 2002; Fernández y Fernández, 2006). El resto se encuentra muy por debajo de esta cifra al registrar matrículas inferiores a los 600 mil estudiantes en ese mismo año.

La TBE en el periodo analizado muestra una tendencia creciente en los cinco países, alcanzándose, en 2003, tasas superiores al 40\% en Chile, Bolivia, Costa Rica y Uruguay, mientras que en México ésta representa el 24\%. Para Trow, los primeros estarían clasificados entre los SES que aplican un modelo de acceso universal (superior al 35\%); el segundo, entre los que cuentan con un modelo de acceso de masas (entre 15\% y el 35\%) (citado en García, 2002).

\section{Diversificación institucional}

La expansión de la matrícula propicia la ampliación de la oferta institucional como una medida para incrementar las oportunidades de acceso. Inicialmente, la región contaba con aproximadamente 75 instituciones (1950), en su mayoría universidades de carácter público, mostrando un crecimiento espectacular a partir de la década de los ochenta y, fundamentalmente, en los noventa. Así, el número de IES latinoamericanas pasa de 5.438, en 1994 -de las cuales 2.515 eran públicas (46\%) y 2.923 privadas (54\%)-, a 7.514 instituciones en 2002, lo que representa un incremento del 38\% respecto de 1994. De éstas, el 65\% corresponde al sector privado (Rama, 2002; García, 2005). 
270 MODELO DE FINANCIACIÓN EN LOS SES DE AMÉRICA LATINA - Susana Fernández, Sara Fernández

En la actualidad, la mayoría de los países latinoamericanos cuenta con SES conformados por instituciones universitarias, responsables de la formación profesional integral (docencia, investigación y difusión de la cultura), y no universitarias, centradas en titulaciones técnicas de corta duración, orientadas al mercado de trabajo. A su vez, en ambos subsistemas coexiste la oferta del sector público y privado ${ }^{3}$, siendo el volumen de la matrícula el que determina el predominio de uno u otro sector. En México, Bolivia y Uruguay, por ejemplo, la matrícula de los SES se concentra en más de un 65\% en las instituciones públicas, mientras que Chile y Costa Rica cuentan con SES predominantemente privados, con más del 55\% en dicho sector (González, 2006).

En la primera década del presente siglo, tal como se muestra en la tabla 2, México registra un total de 1.722 IES, seguido por Chile, con 221, Costa Rica, con 121, y Bolivia y Uruguay, con 51 y 31, respectivamente.

Tabla 2: Estructuración de la oferta de los SES

\begin{tabular}{l|r|r|r|r|r|r|r|r|r|r}
\hline \multirow{2}{*}{ SES } & \multirow{2}{*}{ Año } & \multicolumn{4}{|c|}{ Universitarias } & \multicolumn{4}{|c}{ No universitarias } \\
\cline { 3 - 11 } & & Públicos & & Privados & & Públicos & & Privados & \\
\cline { 3 - 11 } & & $\mathrm{N}^{\circ}$ & $\%$ & $\mathrm{~N}^{\circ}$ & $\%$ & $\mathrm{~N}^{\circ}$ & $\%$ & $\mathrm{~N}^{\circ}$ & $\%$ \\
\hline México(a) & 2002 & 271 & 47 & 306 & 53 & 403 & 35 & 742 & 65 \\
\hline Chile & 2005 & 16 & 25 & 47 & 75 & n.a. & 0 & 158 & 100 \\
\hline Bolivia & 2004 & 15 & 29 & 36 & 71 & No se proporcionan datos \\
\hline Costa Rica & 2004 & 4 & 7 & 50 & 93 & 7 & 10 & 60 & 90 \\
\hline Uruguay & 2003 & 4 & 27 & 11 & 73 & 13 & 81 & 3 & 19 \\
\hline
\end{tabular}

(a) Tomado de Didriksson et al. (2003)

n.a.: No aplica

Fuente: Elaboración propia a partir de Curti (2006).

Para el caso de México, los informes consultados no emplean la denominación "universitaria y no universitaria" para clasificar sus IES, sin embargo, es posible asumir dicha categorización si atendemos al

3 Cuba representa la excepción, ya que su SES está compuesto únicamente por instituciones públicas que ofrecen una formación gratuita, tanto en las titulaciones de grado como de posgrado (Martín, 2003). 
área formativa y a la duración de las titulaciones. Los cinco países, a excepción de Uruguay, han diversificado sus SES, siendo significativo el crecimiento de las instituciones privadas, ya que representan más del $60 \%$ del total de IES, concentradas, en su gran mayoría, en el sector no universitario. Por el contrario, este mismo país cuenta con un número reducido de IES, con un peso similar de ambos sectores.

\section{El esfuerzo financiero}

El esfuerzo financiero que los gobiernos realizan en educación se determina, fundamentalmente, por el porcentaje de PIB que destinan a este servicio. En la tabla 3 se muestra el porcentaje que asignaron a gasto público en educación y en educación superior en 2000 y 2002. Para obtener estas cifras fue necesario recurrir a diversas fuentes de información.

Tabla 3: Gasto público en educación y educación superior como porcentaje del PIB (2000-2002): análisis comparativo

\begin{tabular}{l|c|c|c|c|c|c|c|c|c}
\hline \multirow{2}{*}{ SES } & \multicolumn{3}{|c|}{ Educación } & \multicolumn{3}{c|}{ Educación superior } & \multicolumn{3}{c}{$\begin{array}{c}\text { Educación superior / } \\
\text { presupuesto educativo (\%) }\end{array}$} \\
\cline { 2 - 12 } & 2000 & 2002 & Tendencia & 2000 & 2002 & Tendencia & 2000 & 2002 & Tendencia \\
\hline México & 4,9 & 5,3 & $\uparrow$ & 0,9 & 1,0 & $\uparrow$ & 18,2 & 19 & $\uparrow$ \\
\hline Chile & 4,2 & $4,4(\mathrm{a})$ & $\uparrow$ & 0,6 & $0,4(\mathrm{a})$ & $\downarrow$ & 14,3 & 9 & $\downarrow$ \\
\hline Bolivia & 5,5 & 6,3 & $\uparrow$ & $1,5(\mathrm{~b})$ & 1,6 & $\uparrow$ & $24(\mathrm{c})$ & 25 & $=$ \\
\hline Costa Rica & 4,4 & 5,1 & $\uparrow$ & $0,9(\mathrm{~b})$ & 1,0 & $\uparrow$ & $18(\mathrm{~b})$ & $16(\mathrm{~b})$ & $\downarrow$ \\
\hline Uruguay & 2,8 & 2,6 & $\downarrow$ & 0,6 & 0,6 & $=$ & 20,3 & 23 & $\uparrow$ \\
\hline Media OCDE & 5,2 & 5,4 & $\uparrow$ & 1,2 & 1,3 & $\uparrow$ & 22,3 & 23,2 & $\uparrow$ \\
\hline
\end{tabular}

Corresponde a 2003

Tomado de Rodríguez (2005)

(c) Ministerio de Educación (2004)

$\uparrow$ Crecimiento $\downarrow$ Reducción $\leftarrow$ Estancamiento

Fuente: Elaboración propia a partir de OCDE (2003 y 2005) y UNESCO (varios años).

El esfuerzo financiero de Bolivia en educación (5,5\%) es superior a la media de la Organización para la Cooperación y el Desarrollo Económico (OCDE) (5,2\%), situándose el resto de los países por debajo de dichos valores. Este resultado se atribuye a que 
272 MODELO DE FINANCIACIÓN EN LOS SES DE AMÉRICA LATINA - Susana Fernández, Sara Fernández

Bolivia se encuentra entre los países más pobres de la región ${ }^{4}$. En 2002 todos los países experimentaron un aumento importante en su esfuerzo educativo, a excepción de Uruguay que reduce, aunque de forma moderada, tal esfuerzo.

Si se analizan conjuntamente los dos periodos para los que se dispone de información, mientras México, Bolivia y Costa Rica incrementan su esfuerzo en la financiación pública de la educación superior, en los dos países restantes dicho esfuerzo ha disminuido (Chile) o se ha mantenido estable (Uruguay).

La prioridad de los gobiernos por financiar otras necesidades nacionales, entre ellas la educación básica, explica la escasa participación de la educación superior dentro del presupuesto público de cada país. En todos los países, exceptuando Bolivia, el porcentaje de gasto público educativo destinado a educación superior se encuentra por debajo de la OCDE en ambos años, mostrándose en México, Bolivia y Uruguay un ligero crecimiento entre 2000 y 2002. Chile y Costa Rica exhiben una reducción de tal participación debido a las políticas de recuperación de costes y a la diversificación de los recursos privados a partir de los setenta (Chile) y ochenta (Costa Rica).

En general, este cociente es inferior a la media OCDE en 2000, lo cual demuestra la reorientación de las políticas hacia otros niveles educativos. Neira y Portela (2003) afirman que la ayuda al desarrollo en materia educativa debería reorientarse, ya que, hasta finales de la década de los noventa, se había concentrado en la población urbana y en las enseñanzas superiores.

\section{Financiación de las universidades públicas}

Los recursos que se destinan a las universidades públicas provienen mayoritariamente de transferencias del tesoro público, canalizadas por medio del presupuesto anual de la nación; los de instituciones

4 En 2000 registra el PIB per cápita más bajo de la región, después de Nicaragua (Wolff y Gurría, 2005). 
privadas, de los pagos de matrícula y la venta de servicios educativos. Chile es una excepción a esta práctica en el caso de las universidades privadas tradicionales 5 .

En América Latina, la carencia de recursos públicos que experimentan las universidades públicas a partir de los ochenta y las inequidades en su distribución han generado la necesidad de introducir dos tipos de cambios en sus sistemas de financiación:

- La transformación de los modelos. Los diferentes países proponen mecanismos que faciliten una distribución más equitativa de los recursos públicos, buscando la racionalización del gasto y la eficiencia. De esta forma, incorporan a sus sistemas de financiación mecanismos como las fórmulas y los contratos-programa.

- La diversificación de los recursos. Los gobiernos incentivan a las universidades en la captación de nuevos fondos por las vías del cobro de matrícula, las donaciones y la comercialización de servicios educativos, tales como asesorías, proyectos de investigación, cursos cortos a funcionarios o empresas, entre otros.

A continuación examinaremos en qué medida se han aplicado estas políticas en los países analizados.

\section{Fondos públicos: transformación de los modelos de financiación}

Antes de los noventa, los cinco países utilizaban, prácticamente de forma exclusiva, modelos de financiación no formales o basados en la negociación. En éstos, los fondos son negociados periódicamente entre la administración y la propia universidad. Un rasgo que caracteriza a dichos modelos es la ausencia de un vínculo entre los recursos recibidos por la universidad y las actividades que ésta

5 Por haber sido creadas con anterioridad a 1981, las universidades privadas consideradas tradicionales reciben apoyo estatal, de modo que los recursos públicos recibidos por éstas prácticamente igualan a los de las universidades del sector público (33\% frente al 36,2\%) (OCDE, 2005). 
274 MODELO DE FINANCIACIÓN EN LOS SES DE AMÉRICA LATINA - Susana Fernández, Sara Fernández

desempeña, dificultando el establecimiento de políticas que incentiven la eficiencia y eficacia de la educación superior. Dentro de aquellos basados en la negociación, la modalidad más extendida es el "modelo incrementalista", que toma como base el presupuesto del año anterior y, previa negociación, establece un incremento sobre el mismo.

A partir de los noventa, los gobiernos introducen modelos de financiación formales que pretenden, a partir de una serie de criterios, mejorar la eficiencia, eficacia y equidad en la distribución de los recursos públicos a las universidades. Estos son los modelos basados en fórmulas y en contratos-programa.

La tabla 4 presenta una síntesis de los modelos actuales de financiación de las universidades públicas en los SES analizados.

Tabla 4: Modelos de financiación de las universidades públicas: análisis comparativo

\begin{tabular}{|c|c|c|c|c|c|}
\hline & $\begin{array}{l}\text { MÉXICO } \\
\text { (2003) }\end{array}$ & $\begin{array}{l}\text { CHILE } \\
(2004)\end{array}$ & $\begin{array}{l}\text { BOLIVIA } \\
(2003)\end{array}$ & $\begin{array}{c}\text { COSTA RICA } \\
(2005)\end{array}$ & $\begin{array}{l}\text { URUGUAY } \\
(2000)\end{array}$ \\
\hline $\begin{array}{l}\text { Financiación basada } \\
\text { en la negociación: } \\
\text { 1. Incrementalista } \\
\text { 2. Porcentaje } \\
\text { determinado }\end{array}$ & $\begin{array}{l}\text { 1. Subsidio } \\
\text { Ordinario } \\
(92 \%)\end{array}$ & $\begin{array}{l}\text { 1. Presupuesto } \\
\text { Base }(80 \%)\end{array}$ & $\begin{array}{c}\text { 1. Subvención Ordinaria } \\
(54 \%) \\
\text { 2. Coparticipación } \\
\text { Tributaria }(39 \%)\end{array}$ & $\begin{array}{l}\text { 1. Fondo } \\
\text { Institucional } \\
\text { (99\%) }\end{array}$ & $\begin{array}{l}\text { 1. Aporte } \\
\text { Presupuestal } \\
\quad(89 \%)\end{array}$ \\
\hline \multirow[t]{2}{*}{$\begin{array}{l}\text { Financiación basada } \\
\text { en fórmulas: } \\
\text { inputs y ouputs }\end{array}$} & \multirow[t]{2}{*}{$\begin{array}{l}\text { Programa } \\
\text { Integral de } \\
\text { Fortalecimiento } \\
\text { Institucional } \\
\text { (PIFI) (5\%) }\end{array}$} & \multirow{2}{*}{$\begin{array}{c}\mid \begin{array}{c}\text { Distribución } \\
\text { basada en el } \\
\text { desempeño } \\
(4 \%)\end{array} \\
\text { Aporte Fiscal } \\
\text { Indirecto (AFI) (11\%) }\end{array} \mid$} & \multirow{3}{*}{\begin{tabular}{|c} 
Subvención extraordinaria \\
para necesidades \\
especíicas $(6 \%)$
\end{tabular}} & \multirow{3}{*}{$\begin{array}{c}\text { Fondo del } \\
\text { sistema } \\
(1 \%)\end{array}$} & \multirow{3}{*}{\begin{tabular}{|c|} 
Aporte \\
Extrapresupuestal \\
$(9 \%)$
\end{tabular}} \\
\hline & & & & & \\
\hline \multirow[b]{2}{*}{$\begin{array}{l}\text { Financiación basada } \\
\text { en contratos } \\
\text { programas }\end{array}$} & \multirow[b]{2}{*}{$\begin{array}{c}\text { Fondo de } \\
\text { Aportación } \\
\text { Múltiple (FAM) } \\
(3 \%)\end{array}$} & \multirow[b]{2}{*}{$\begin{array}{c}\text { Fondo de Desarrollo } \\
\text { Institucional y } \\
\text { MECESUP }(5 \%)\end{array}$} & & & \\
\hline & & & $\begin{array}{c}\text { Fondo de Mejoramiento } \\
\text { de la Calidad } \\
\text { (FOMCALIDAD) (1\%) }\end{array}$ & & \begin{tabular}{|} 
Partidas concursables \\
(proyectos \\
investigación) \\
$(2 \%)$
\end{tabular} \\
\hline
\end{tabular}

Los países del estudio, excepto Chile, utilizan modelos de financiación no formales o basados en la negociación, fundamentalmente el incrementalista, para asignar el 90\% de los recursos públicos a las actividades básicas de las universidades. Dichos recursos se entregan sobre la base del número de estudiantes (Bolivia 
y Costa Rica), de docentes (México) o de los gastos asociados a los programas académicos (Costa Rica y Uruguay); en todo caso, se trata de criterios más relacionados con los inputs que con los outputs de las universidades.

Bolivia emplea, además, otro modelo de financiación no formal, en el cual la administración define un porcentaje fijo sobre los ingresos nacionales ${ }^{6}$ (coparticipación tributaria). Chile, por el contrario, asigna mediante el sistema incrementalista el 80\% de los recursos públicos, adoptando un esquema diferente al considerar como presupuesto base el 95\% del asignado en el año anterior y el 5\% que depende del desempeño institucional, siendo fundamental la capacidad de las universidades en la captación de esta segunda categoría de fondos para mantener su presupuesto.

Además, los cinco países utilizan la financiación mediante fórmulas y contratos-programa para entregar recursos públicos a las universidades. Por esta segunda vía, Chile asigna hasta un 20\% de sus fondos públicos; el resto como máximo lo hace en un 11\%.

Inicialmente, los modelos de financiación basados en fórmulas asignaban recursos mediante mecanismos no competitivos, fundamentalmente en función de inputs (insumos). Luego adoptaron otros criterios, asignándolos de forma competitiva o basados en outputs (resultados). Chile es un claro ejemplo, ya que las universidades compiten por los fondos del Aporte Fiscal Indirecto (11\% de los recursos públicos), cuya asignación depende de su capacidad para captar a los estudiantes de educación media con los mejores resultados académicos ${ }^{7}$ en la Prueba de Selección Universitaria (PSU) (outputs). De la misma forma, México contempla en su Proyecto Integral

6 La Ley de Participación Popular establece que el 5\% de los ingresos nacionales sea destinado a la educación superior y se distribuya entre las universidades públicas autónomas en función de la población en la que se encuentran ubicadas.

7 Se considera un total de 27.500 estudiantes que se clasifican en cinco tramos en función de su nota (5.500 estudiantes), de forma que las universidades que logren atraer a los estudiantes con notas más altas (último tramo) recibirán mayores recursos (Fernández y Fernández, 2005). 
276 MODELO DE FINANCIACIÓN EN LOS SES DE AMÉRICA LATINA - Susana Fernández, Sara Fernández

de Fortalecimiento Institucional (PIFI) -el cual supone para las universidades un $5 \%$ de los fondos públicos- diversos mecanismos que asignan recursos de forma competitiva, como el Programa de Mejoramiento del Profesorado y el Fondo para la Modernización de la Educación Superior.

Los contratos-programa, que en general canalizan menos del $5 \%$ de los recursos públicos a las universidades, suelen ser, en su mayoría, de carácter concursable, como el Fondo de Aportación Múltiple, en México; el Fondo de Mejoramiento de la Calidad, en Bolivia; el Fondo de Desarrollo Institucional y el Programa para el Mejoramiento de la Calidad de la Educación Superior, en Chile, o las partidas que destina Uruguay a proyectos de investigación.

No ha sido posible establecer la forma de distribución de los porcentajes que se enmarcan en la zona gris de la tabla 4 , ya que se carece de información respecto del modelo de financiación utilizado (fórmulas o contratos-programa) o del carácter de dichos recursos (competitivos o no competitivos).

La consideración de las fórmulas y los contratos-programa en los cinco países constituye una respuesta a la necesidad de aumentar los recursos e introducir mayor eficiencia en el gasto público. Sin embargo, dada su reciente incorporación, los fondos que se distribuyen mediante estos esquemas son aún muy reducidos, predominando el modelo incrementalista.

\section{Fondos privados: diversificación de los recursos}

Si bien la mayoría de los recursos de las instituciones proviene de fondos públicos, éstos resultaron insuficientes para atender la expansión del sistema. Por ello, los gobiernos pusieron en marcha medidas encaminadas a incentivar la generación de fondos adicionales por parte de las universidades públicas a través de cobros de matrícula, captación de donaciones y venta de servicios educativos. Sin embargo, pese a los esfuerzos por incrementar estos ingresos, su participación se reduce prácticamente al cobro de matrículas. 
En países como México, Chile, Bolivia y Costa Rica dichas políticas fueron aprobadas e introducidas a partir de la década de los setenta, después de los constantes esfuerzos por persuadir a la población estudiantil y a la sociedad en general de la necesidad de mayores recursos para asegurar la equidad y la calidad del sistema. En Uruguay, la educación superior se resiste a esta medida en defensa del derecho a la gratuidad de la educación pública, siendo mínimo o casi inexistente el esfuerzo dirigido a la captación de recursos privados (el 100\% de los recursos propios del SES uruguayo proviene de la venta de servicios). En México, Bolivia y Costa Rica los ingresos provenientes del pago de matrículas representan más del 70\% de los recursos propios, mientras que en Chile representan prácticamente la totalidad de ellos (98\%).

La tabla 5 muestra el esfuerzo realizado por los cinco países en la generación de recursos propios ${ }^{8}$.

Tabla 5: Porcentaje de financiación pública vs. financiación privada a las universidades públicas (2002): análisis comparativo

\begin{tabular}{l|c|c}
\hline & Público (\%) & Privado (\%) \\
\hline México(a) & 71 & 29 \\
\hline Chile(a)* & 17 & 83 \\
\hline Bolivia(b) & 81 & 19 \\
\hline Costa Rica & 85 & 15 \\
\hline Uruguay(a) & 96,9 & 3,1 \\
\hline Media OCDE & 78,6 & 21,4 \\
\hline
\end{tabular}

*Corresponde a 2003

a) OCDE; Education at a Glance 2005

b) Se excluyen recursos que no tienen un origen claro (disminución de otros activos financieros como movimientos de caja y bancos, disminución de cuentas por cobrar, entre otros)

Fuente: Elaboración propia a partir de OCDE (2003b) y Rodríguez (2005).

8 Estos datos no siempre son comparables, ya que la información de Costa Rica y Bolivia procede del documento de Rodríguez (2005), elaborado para el IESALC, mientras que las cifras aportadas por la OCDE para México, Chile y Uruguay pueden estar sobrevalorados al incluir los subsidios públicos a los hogares. 
278 MODELO DE FINANCIACIÓN EN LOS SES DE AMÉRICA LATINA - Susana Fernández, Sara Fernández

En México, Bolivia, Costa Rica y Uruguay las universidades dependen de la financiación pública en más de un $70 \%$, mientras que en Chile la provisión de fondos es predominantemente privada, superando en casi cinco veces los recursos públicos y representando el $83 \%$ de la provisión total de las universidades tradicionales. Ello responde a las estrategias de diversificación de los fondos impulsada en la década de los setenta, anticipándose al resto de países donde se dieron una década después (Fernández y Fernández, 2005).

Tanto en México como en Chile, los fondos privados alcanzan una participación importante en la financiación de las IES públicas, permitiéndoles contar con un mayor margen de maniobra en la gestión institucional. Ello es beneficioso porque, en general, la excesiva dependencia de recursos públicos puede generar inestabilidad financiera; se trata de una región donde, con escasas excepciones, los países tienen una estructura fiscal débil, lo cual se refleja año tras año en la asignación y giro de recursos a las IES, dificultando su desempeño normal y no permitiendo el diseño y ejecución de planes a mediano y largo plazo. Esta situación no se da en los demás países analizados; en Uruguay, por ejemplo, la política de gratuidad en el acceso a la educación superior reduce las posibilidades para incrementar dichos recursos por la vía de la venta de servicios educativos.

\section{Conclusiones}

El análisis de las reformas en los modelos de financiación ha permitido encontrar elementos comunes en los SES analizados, ya que todos han pasado por los mismos procesos aunque no de forma simultánea. Como se indicó, dichas reformas se han centrado sobre todo en dos estrategias: a) la transformación de los modelos de financiación y 2) la diversificación de los recursos.

A pesar de que cada SES había sido clasificado por Fernández y Fernández (2007) en grupos diferentes, es posible concluir que no existen diferencias significativas en los modelos de financiación, ya que todos utilizan modelos basados en la negociación, fundamentalmente 
el incrementalista, para asignar el 90\% de los recursos públicos a las actividades básicas de las universidades. Chile es la excepción, porque canaliza a través del modelo incrementalista el $80 \%$ de los recursos públicos, lo que indica un esfuerzo importante en el empleo de mecanismos basados en fórmulas y en contratos-programa, que buscan mejorar la eficiencia en la asignación de la financiación pública. En el resto de los países, la adopción de estos mecanismos es relativamente reciente, resultando difícil establecer, por falta de información, su naturaleza (fórmulas o contratos-programa) y principales características (competitivos o no competitivos) (recuérdese la zona gris de la tabla 4).

Tampoco hay diferencias en los mecanismos que incentivan la captación de recursos privados por parte de las instituciones, ya que en su mayoría proceden de los cobros por matrícula. Chile vuelve a ser la excepción, ya que se ha anticipado en dichos cobros en las instituciones públicas (1980), lo cual explica su peso en la financiación privada, con una clara orientación al mercado en el cual las instituciones compiten para ofrecer servicios educativos. En el resto de los países, estos cobros fueron introducidos recientemente como una solución al crecimiento de la matrícula y a la escasez de los recursos públicos, lo cual supuso serios conflictos al tratar de persuadir a la sociedad de la necesidad de contribuir a financiar su formación. En estos SES, los ingresos por medio de cobros no suponen cantidades elevadas e incluso en el uruguayo no se ha aplicado esta política, en defensa de la gratuidad de la educación superior.

Por tanto, con independencia del grupo de pertenencia de los SES analizados, no existen diferencias en los modelos de financiación utilizados. Los cambios en dicho modelo pueden corregir algunos de los aspectos negativos que se derivan de las políticas macroeconómicas. Tal como se expuso, la mayoría de estos países realizaban un escaso esfuerzo en la financiación de sus SES, ya fuera por la escasez de recursos (Bolivia), por la prioridad que otorgan a otros niveles educativos (México, Chile, Costa Rica) o por ambos motivos. 
280 MODELO DE FINANCIACIÓN EN LOS SES DE AMÉRICA LATINA - Susana Fernández, Sara Fernández

No obstante, con independencia del volumen de recursos públicos que se destina a la educación superior, una cuestión fundamental es cómo se distribuyen entre las universidades. El modelo incrementalista, ampliamente empleado por los países analizados, no es el mecanismo más adecuado para mejorar la calidad de la educación superior, ya que la ausencia de un vínculo directo entre los fondos asignados y las actividades desempeñadas por las universidades imposibilita la aplicación de políticas coherentes con el aumento de la eficiencia y de la eficacia. Otro de los inconvenientes de dicho modelo es que el monto final de los recursos se define mediante un proceso de negociación entre las partes, lo que supone una alta dosis de subjetividad y escasa transparencia.

Por ello, cabría esperar que en los próximos años los países acrecentasen los recursos públicos utilizando modelos de financiación basados en fórmulas y en contratos-programa, con el fin de mejorar el funcionamiento de las instituciones y, con ello, la calidad de la educación superior.

\section{Referencias bibliográficas}

Barquero, J.; Calvo, E.; Ortiz, M. et al. (2006) Financiamiento de la educación superior: antecedentes y tendencias en el contexto nacional e internacional. San José: Editorial de la Universidad de Costa Rica.

Brunner, J. J. (1994) Educación superior en América Latina: Una agenda de problemas, politicas y debates en el umbral del año 2000. Colombia: Proyecto de Políticas Comparadas de Educación Superior.

CONARE. (2004) La nueva visión de la educación superior universitaria estatal. San José: CONARE.

Conejo, C. (2004) Financiamiento de la educación superior en América Latina: el caso de Costa Rica. Caracas: Observatorio Digital para la Educación Superior en América Latina y el Caribe, IESALC/UNESCO, pp. 4-24.

Curti, C. (2006) Estudio diagnóstico: estadísticas de educación superior en América Latina y el Caribe. En: Rama, C. (ed.) Informe sobre la educación superior en América Latina y el Caribe 2000 - 2005: la metamorfosis de la educación superior. Caracas: Editorial Metrópolis C. A., pp. 233-240. 
Fernández, S. y Fernández, S. (2004) Financiación de la educación en América Latina: Argentina, México y Brasil. En: Grao, J. (coord.) Actas de las XIII Jornadas de la AEDE. San Sebastián: Universidad de País Vasco, pp. 353-360.

Fernández, S. y Fernández, S. (2005) La transformación del sistema de educación superior chileno. En: García, M. M.; González, M. C. y Muniz, M. A. (coords.) Actas de las XIV Jornadas de la AEDE. Oviedo: Universidad de Oviedo, pp. 417-429.

Fernández, S. y Fernández, S. (2007) El esfuerzo financiero público: una variable discriminante para los sistemas de educación superior latinoamericanos. Revista de la Educación Superior, XXXVI (1), (141). Próxima publicación.

García, C. (2002) Desafíos emergentes y tensiones acumuladas. Educación superior en América Latina. En: Casanova, H. (coord.) Nuevas políticas de educación superior. La Coruña: Netbiblo, pp. 891-127.

García, C. (2005) Financiamiento de la educación superior en América Latina. En: GUNI/UNESCO. Educación superior en el mundo 2006. El financiamiento de las universidades. Madrid, Barcelona, México: Ediciones Mundi-Prensa, pp. 3-36.

González, H. (2006) Estadísticas de la educación superior. En: Rama C. Informe sobre la educación superior en América Latina y el Caribe 2000 - 2005: la metamorfosis de la educación superior. Caracas: Editorial Metrópolis C. A., pp. 240-261.

Martín, E. (2003) Informe nacional sobre educación superior en Cuba. Caracas: Observatorio Digital para la Educación Superior en América Latina y el Caribe, IESALC/UNESCO, pp. 14-20.

Ministerio de Educación (2004) La educación en Bolivia: indicadores, cifras y resultados. La Paz: Dirección de Análisis.

OCDE. (2003) Regards sur l'éducation. les indicateurs de l'OCDE, Education at a glance. Paris: OCDE.

OCDE. (2005) Regards sur l'éducation. les indicateurs de l'OCDE, Education at a glance. Paris: OCDE.

Psacharopoulos, G. y Patrinos, H. A. (2002) Returns to Investment in Europa: A Further Update. Policy Research Working Paper, (2881), pp. 1-28.

Rama, C. (2002) La tercera reforma de la educación superior en América Latina. Agenda Académica, 9, (1-2), pp. 1-38. 
282 MODELO DE FINANCIACIÓN EN LOS SES DE AMÉRICA LATINA - Susana Fernández, Sara Fernández

Rodríguez, F. (2005) El financiamiento de la educación superior en América Latina: una visión panorámica. En: Rama C. Informe sobre la educación superior en América Latina y el Caribe 2000 - 2005: la metamorfosis de la educación superior. Caracas: Editorial Metrópolis C. A., pp. 71-78.

UNESCO. (2003) Compendio Mundial de la Educación 2003: Comparación Estadística de la Educación en el Mundo. UNESCO.

UNESCO. (2004) Compendio Mundial de la Educación 2004: Comparación Estadística de la Educación en el Mundo. UNESCO.

UNESCO. (2005) Compendio Mundial de la Educación 2005: Comparación Estadística de la Educación en el Mundo. UNESCO.

UNESCO. (2006) Compendio Mundial de la Educación 2006: Comparación Estadística de la Educación en el Mundo. UNESCO.

Wolff, L. y Gurría, M. (2005) Money counts: Projecting Education Expenditures in Latin American and the Caribbean to the year 2015. Montreal: Institute for Statistics UNESCO.

Recibido: 14 de junio de 2007

Aceptado: 12 de septiembre de 2007 